\title{
Design of a complex filter for depth of focus extension
}

\author{
Frédéric Diaz, ${ }^{1,2, *}$ François Goudail, ${ }^{2}$ Brigitte Loiseaux, ${ }^{1}$ and Jean-Pierre Huignard ${ }^{1}$ \\ ${ }^{1}$ Thales Research \& Technology, RD 128, 91767 Palaiseau Cedex, France \\ ${ }^{2}$ Laboratoire Charles Fabry de l'Institut d'Optique, CNRS, Université Paris-Sud, Campus Polytechnique, RD 128, \\ 91127 Palaiseau Cedex, France \\ *Corresponding author: frederic.diaz@thalesgroup.com
}

Received October 23, 2008; revised February 10, 2009; accepted March 5, 2009; posted March 13, 2009 (Doc. ID 103148); published April 7, 2009

\begin{abstract}
Different methods such as axilens and binary-phase filter have been investigated to improve the depth of focus. A method is proposed to calculate an amplitude-phase pupil filter and obtain the desired distribution of intensity along the optical axis. It produces a narrow spot with a uniform intensity level over a large depth of focus, comparable to the performance obtainable with binary-phase filters. This filter is of particular interest for applications where very low intensity fluctuations along the focus range are required. () 2009 Optical Society of America
\end{abstract}

OCIS codes: $070.2580,090.1760,100.1390$.

The design of pupil filters that enhance the depth of focus (DOF) of optical systems is a topic of great interest, and the simplest ones are probably amplitude filters [1]. Continuous phase filters [2-4] and binaryphase filters [5-7] can also enhance the DOF. They are usually considered as energy efficient compared to amplitude filters. However, in DOF extension the relevant parameter is not the total transmitted energy, but the "useful" one, that is, the energy concentrated in the focal line. With this criterion, amplitude-phase (complex) filters may have better performance. Besides, some complex filters have already been designed [8-10].

Our goal is to produce a light distribution as uniform as possible along the focal line. We will define the DOF as the length of the region along the optical axis, where the amplitude distribution has fluctuations lower than a given threshold. For given DOF and allowed fluctuations, our purpose is to determine the filter that maximizes the mean intensity in the DOF region. To reach this goal, we will compare two types of methods: a classical one based on binaryphase filter (BPF), also used for superresolution [11] and creation of circular Dammann gratings [12], and a new approach based on a holographically generated complex filter (HGCF).

The BPF is composed of an array of $n$ concentric rings whose phases are alternatively zero and $\pi$. Their outer radii are denoted $r_{1}, r_{2}, \ldots, r_{n-1}$ and are normalized with respect to the pupil radius [Fig. 1(a)]. Assuming that the aperture is small compared to the focal length, we obtain the following normalized amplitude distribution near the optical axis generated by a lens and a BPF:

$$
\begin{aligned}
A(\rho, z)= & \exp (j k z) / z \times \exp \left(j k \rho^{2} / 2 z\right) \\
& \times \int_{0}^{R} P\left(\rho_{P}\right) \rho_{P} J_{0}\left(k \rho \rho_{P} / z\right) \\
& \times \exp \left[j k \rho_{P}{ }^{2}(1 / z-1 / f) / 2\right] \mathrm{d} \rho_{P},
\end{aligned}
$$

where $\lambda$ is the wavelength, $k=2 \pi / \lambda ; f$ is the focal length; $R$ the radius of the lens; $\rho$ and $z$ are the transverse and axial coordinates; $\rho_{P}$ is the transverse coordinate in the pupil plane; $J_{0}$ is the zero-order Bessel function; and $P\left(\rho_{P}\right)$ is the complex amplitude of the BPF, equal to \pm 1 .

Using Eq. (1), one can find parameters of the BPF that generate the desired DOF. We performed an exhaustive search by computing the DOF and mean intensity in the DOF region of all three-, five-, and seven-ring BPFS generated by varying the radii of each ring with steps of $0.001,0.005$, and 0.01 , respectively. For each value of the DOF, we kept the BPF that gave the largest mean intensity. The mean intensity versus the DOF is shown in Fig. 1(b), using a lens with a focal length of $800 \mathrm{~mm}$ and an aperture of $16 \mathrm{~mm}$, at a wavelength of $1064 \mathrm{~nm}$, with variations below $10 \%$. With a three-ring $\mathrm{BPF}$, one can achieve a maximal DOF of $42 \mathrm{~mm}$. Interestingly, for lower DOF, the use of five and seven rings does not improve the mean intensity. However, increasing the number of rings extends the range of accessible DOF.

As an original alternative we now propose to design a complex filter with the classical approach used for computer-generated holograms. Such a design first specifies the desired normalized amplitude at chosen points along the optical axis and then com-
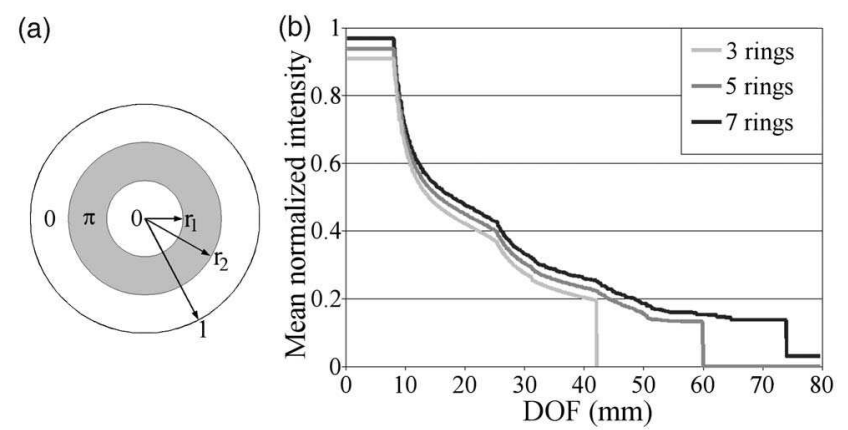

Fig. 1. (a) Structure of the BPF. (b) Mean normalized intensity in the DOF region versus DOF for BPFs with three, five, and seven rings. Curves are vertically shifted for clarity. 
putes the filter that generates this distribution. That is to say, we can directly generate a complex filter that has a given DOF that we will call an HGCF.

Let us consider $n$ points situated at the coordinates $z_{i}, i \in[1, n]$ on the optical axis, uniformly distributed throughout the desired DOF region, and set the desired normalized amplitude at each point equal to $D_{i}$. To obtain low variations inside the DOF region, we first set $D_{i}=1$. To calculate the HGCF providing the normalized amplitudes $D_{i}$ at the coordinates $z_{i}$, we consider these points as elementary radiating point sources that emit waves of amplitudes $A_{i}$. Each point source generates at the pupil plane a complex amplitude $T_{i}(\rho)=A_{i} \exp \left(-j k \rho^{2} / 2 z_{i}\right) / z_{i}$, which can be seen as the complex transmittance of a lens with a focal length $z_{i}$. Illuminated by a plane wave, this lens generates an amplitude distribution along the optical axis equal to

$$
A_{z}\left(z, A_{i}, z_{i}\right)=A_{i} \times A_{p t}\left(z, z_{i}\right) \times \exp \left(j \varphi_{p t}\left(z, z_{i}\right)\right),
$$

with $A_{p t}\left(z, z_{i}\right)=2 \sin \left[k\left(1 / z-1 / z_{i}\right) R^{2} / 4\right] /\left[k\left(z_{i}-z\right)\right]$ and $\varphi_{p t}\left(z, z_{i}\right)=k\left(z-z_{i}\right)+k\left(1 / z-1 / z_{i}\right) R^{2} / 4$.

The coherent combination of the point sources generates at the pupil plane a complex filter of transmittance $T(\rho)=\Sigma_{i} T_{i}(\rho)$, which corresponds to the amplitude distribution $A_{z \text { tot }}(z)=\Sigma_{i} A_{z}\left(z, A_{i}, z_{i}\right)$. The coefficients $A_{i}$ so that $\forall i, A_{z \text {,tot }}\left(z_{i}\right)=D_{i}$, are calculated by solving the following set of linear equations:

$$
\vec{D}=Z \times \vec{A}
$$

where vector $\vec{D}$ contains the $D_{i}$, vector $\vec{A}$ contains the $A_{i}$, and $Z$ is a $n \times n$ matrix containing the amplitude associated to each point source $i$, with $A_{i}=1$, at the coordinates $z_{j}: Z_{i, j}=A_{z}\left(z_{j}, 1, z_{i}\right)$. The values of $A_{i}$ are obtained by inverting the matrix $Z$, and the complex transmission of the obtained HGCF is

$$
T(\rho)=\frac{\sum_{i} T_{i}(\rho)}{\max _{\rho}\left|\sum_{i} T_{i}(\rho)\right|} \exp \left(j k \rho^{2} / 2 f\right),
$$

where the phase already introduced by the lens has been removed and the transmittance normalized so that its maximal modulus is 1 . Since we consider spatially incoherent imaging, the interesting value is the intensity distribution along the optical axis,

$$
I_{z}(z) \propto\left|\sum_{i} A_{i} \times A_{p t}\left(z, z_{i}\right) \times \exp \left(j \varphi_{p t}\left(z, z_{i}\right)\right)\right|^{2} .
$$

While the function $A_{p t}\left(z, z_{i}\right)$ has relatively slow variations, the $\exp \left(j \varphi_{p t}\left(z, z_{i}\right)\right)$ phase terms introduce fast and large intensity fluctuations between the point sources. These terms can be removed by choosing point sources satisfying the relation $\varphi_{p t}\left(z_{i}, f\right)=2 \pi p_{i}$ with $p_{i}$ integer numbers. By taking point sources close enough to each other compared to the width of the function $A_{p t}\left(z, z_{i}\right)$, the variations of intensity can be reduced below $1 \%$. The mean intensity in the DOF region can be further improved by optimizing their positions $z_{i}$ and desired amplitudes $D_{i}$. We also use two extra point sources, before and after the DOF region, with low desired amplitudes, to decrease faster the intensity outside the DOF region. We noticed that this also increases the intensity in the DOF region. Performing a numerical optimization, we managed to increase the intensity in the DOF region up to a factor of 3 compared to the nonoptimized HGCF.

Figure 2 illustrates an example of results with seven point sources in the DOF region and two outside to obtain a $33 \mathrm{~mm}$ DOF, which represents a defocus of $W_{20}=0.76 \lambda$, with fluctuations below $1 \%$, centered on the focal plane. The FWHM of the pointspread function (PSF) of the HGCF should be compared to that of a single lens, which exhibits only a $2.1 \mathrm{~mm}$ DOF with fluctuations below $1 \%$. This represents a gain on the $\mathrm{DOF}$ of a factor of 16 , while the maximal intensity obtained with the HGCF on the axis is divided by a factor of 8 compared to the single lens at the focal plane.

We compared in Fig. 2 the HGCF with the BPF having the same DOF at the same fluctuation level, whose radii are $r_{1}=0.24, r_{2}=0.31, r_{3}=0.39, r_{4}=0.47$, $r_{5}=0.84$, and $\mathrm{r}_{6}=0.96$. Their performances are comparable, although the HGCF has a mean amplitude transmission of $63 \%$. This drop of transmission is compensated by a better concentration of energy in the DOF region, thanks to the contribution of the amplitude part of the filter. Moreover, with the HGCF, it is possible to achieve an accurate design of the DOF region that is directly linked to the choice of point sources. In our example, the DOF region is centered on the focal point, whereas the DOF region of the $\mathrm{BPF}$ is asymmetrically distributed between $z=768$ and $801 \mathrm{~mm}$. Further advantages of the HGCF are the sharper decrease of the intensity at the limit of the DOF region, lower sidelobes (about 16\% of the intensity in the DOF region for the HGCF against up to $23 \%$ for the BPF), and the better invariance of the transverse spot size with respect to $z$ inside the DOF region. The phase distributions of the $\mathrm{BPF}$ and the HGCF plotted in Fig. 3 show great similarities. Despite them, setting the transmission of the HGCF to 1 on the whole pupil does not provide good results. The amplitude modulation is required to yield an optimal result.

In conventional optically generated off axis holograms, the spacing of the interference fringes is comparable to the wavelength, making the hologram output very sensitive to wavelength. With the HGCF, since we operate along the direction of the zero order
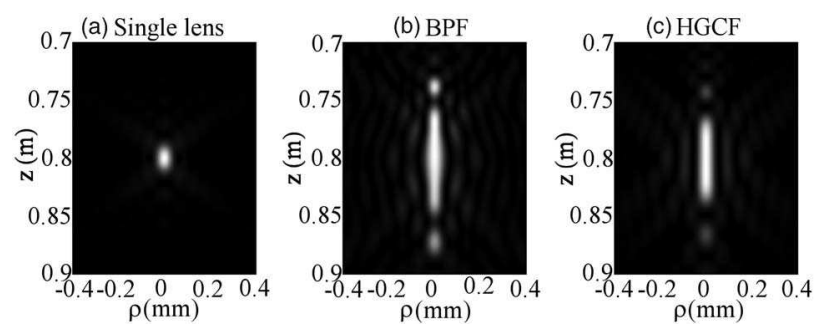

Fig. 2. (a) Normalized intensity distribution in the focal region for the single lens, (b) for the BPF with a DOF of $33 \mathrm{~mm}$ with fluctuation below $1 \%$, and (c) for the HGCF with the same DOF. 


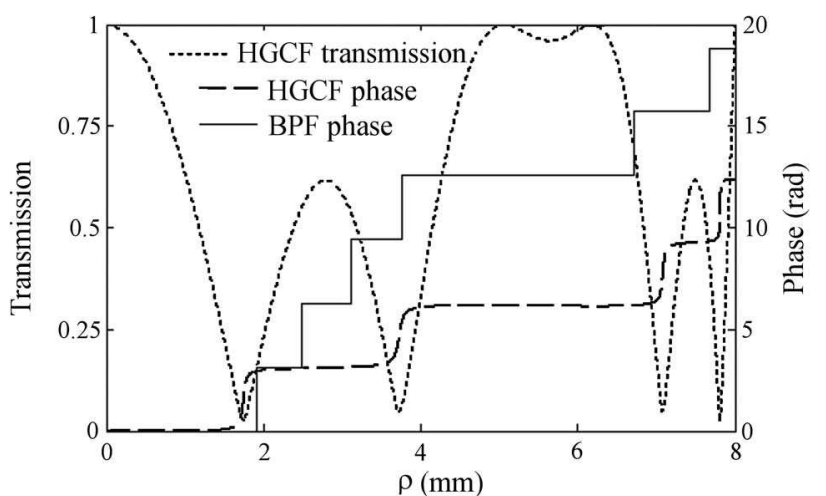

Fig. 3. Profiles of the amplitude transmission and phase of the BPF and the HGCF.

of diffraction, the scale of the spatial variations of the phase and the amplitude is very large compared to the wavelength, as can be seen in Fig. 3. Consequently, assuming that the medium where the phase modulation is recorded is nondispersive, we calculated that its performance remains fairly constant for an illumination with a bandwidth of around $100 \mathrm{~nm}$. The behavior of the HGCF with respect to wavelength is comparable to that of the BPF.

Finally, we compared in Fig. 4 the mean intensities of the optimal BPF and HGCF versus the DOF, for

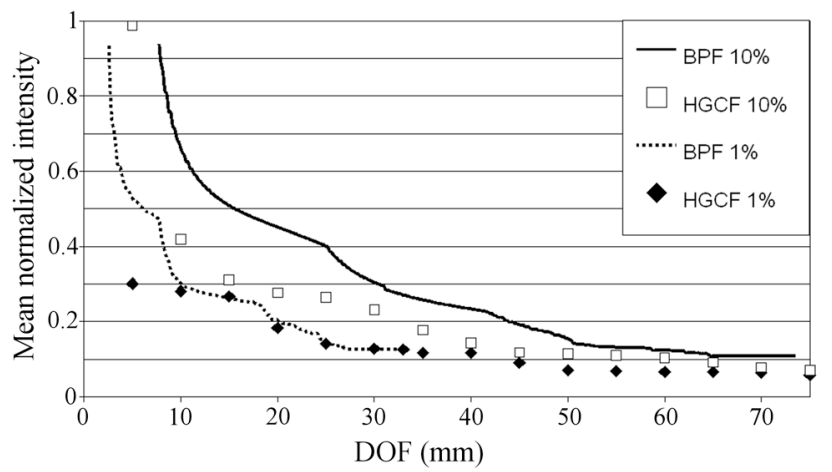

Fig. 4. Mean normalized intensity versus DOF for BPF and HGCF with fluctuations below $10 \%$ and $1 \%$. fluctuations below $10 \%$ and $1 \%$. We can point out that, for DOF intensity fluctuations below $10 \%$, the $\mathrm{BPF}$ is clearly more efficient than the HGCF. However, for fluctuations below $1 \%$, the HGCF yields results similar to the seven-ring BPF, while it makes it possible to reach a longer DOF. We voluntarily limited ourselves to seven rings for the BPF, and we did not find any configuration giving a DOF larger than $33 \mathrm{~mm}$.

We proposed a new holographic design for an amplitude phase filter that we called HGCF. Such a filter can be realized using nanostructured materials for the phase and variable transmission mask for the amplitude. The $\mathrm{HGCF}$ is an alternative to the $\mathrm{BPF}$ for applications where very low fluctuations of the amplitude distribution along the optical axis are required and when the shape of the focal line has to be precisely tailored. It also benefits from lower sidelobes and a better invariance of the PSF with respect to $z$ inside the DOF region. Moreover, it is as energy efficient as a BPF, even taking into account its absorption.

\section{References}

1. W. T. Welford, J. Opt. Soc. Am. A 50, 749 (1960).

2. J. H. McLeod, J. Opt. Soc. Am. 44, 592 (1953).

3. J. Sochacki, S. Bara, Z. Jaroszewicz, and A. Kolodziejczyk, Opt. Lett. 17, 7 (1992).

4. E. Marom, D. Mendlovic, N. Konforti, J. Katz, and C. Tan, Proc. SPIE 3010, 30 (1997).

5. H. Wang and F. Gan, Appl. Opt. 40, 5658 (2001).

6. L. Liu, C. Liu, W. C. Howe, C. J. R. Sheppard, and N. Chen, Opt. Lett. 32, 2375 (2007).

7. L. Liu, F. Diaz, L. Wang, B. Loiseaux, J.-P. Huignard, C. J. R. Sheppard, and N. Chen, J. Opt. Soc. Am. A 25, 2095 (2008).

8. M. Yun, L. Liu, J. Sun, and D. Liu, J. Opt. Soc. Am. A 22, 272 (2005).

9. S. Förster, H. Gross, F. Höller, and L. Höring, Proc. SPIE 5962, 44 (2005).

10. S. Bagheri and B. Javidi, Opt. Lett. 33, 757 (2008).

11. J. Jia and C. Zhou, and L. Liu, Opt. Commun. 228, 271 (2003).

12. C. Zhou, J. Jia, and L. Liu, Opt. Lett. 28, 2174 (2003). 